\title{
Children and the History of Architecture
}

\section{- (A Reflecting Consideration towards the Quality of Children's Familiarity with the Instructions of the History of Architecture)}

\author{
Azin Ehteshami \\ Iranian Architecture, Architecture and Planning Department of Isfahan University of Art, Isfahan, Iran \\ Email: azin.ehteshami68@gmail.com
}

How to cite this paper: Ehteshami, A. (2018). Children and the History of Architecture. Creative Education, 9, 1287-1301. https://doi.org/10.4236/ce.2018.99096

Received: May 11, 2018

Accepted: July 7, 2018

Published: July 10, 2018

Copyright $\odot 2018$ by author and Scientific Research Publishing Inc. This work is licensed under the Creative Commons Attribution International License (CC BY 4.0).

http://creativecommons.org/licenses/by/4.0/

\begin{abstract}
The present article is an introduction on identifying the quality of children's confrontation with the history of architecture, in which is planned to respond to the question regarding the type of knowledge in the field of the history of architecture that children can learn. Thus, the viewpoints of the scholars in the field of child growth have primarily been dealt with regards to the mental ability of children in learning various subjects. By analyzing the viewpoints for different ages of the children, it has been tried to identify and extract the capacities that are related to the instructions of the architectural history. Thus, 11 cases of the related capabilities are introduced in this paper. The compatibility of each capacity with the perception of the history of architecture for the children is discussed for each case. Moreover, the other considered point for each section is the use of each one of the capacities for the children or the community.
\end{abstract}

\section{Keywords}

History of Architecture, Child, Architectural History Instruction, Architectural History Perception

\section{Introduction}

Placing the children among the addressees of the history of architecture is an important issue that its importance can be analyzed from various aspects, but the aim of the present study is not the necessity and importance of that issue, and it is dealing with the subject of children in identifying and recognizing the history of architecture. According to the subject of the study, considering the subject has two different aspects: the first aspect is the consideration of the intellectual capacities of children and analyzing what intellectual capacities the 
children have in confronting with the phenomena such as architecture and the history of architecture. The second aspect is finding the relation between the intellectual capabilities and the cognition world of a phenomenon such as architecture. However, the important point in this study is that the two aspects are not considered separately; i.e. from the beginning and through recognizing the intellectual capacities of children, the capacities are selected for the analysis that has even implicit relations with architectural cognition and its history. Hence, all the herewith considered points in introducing the intellectual capabilities of children are somewhat related to the field of architectural knowledge.

In addition to the ideas quoted by Western scholars, studies have also been conducted on children of this age in Iran. In the categories available in some of these studies, children of different age groups are classified with the class they are studying. Our children are more likely to be placed in the fourth and fifth grades of the elementary school or slightly higher and lower. In these studies, students of each class (each age group) are subject to field research, the results of which are some it reveals other characteristics of this age group.

\section{Literature Reviews}

\section{Capacities of memorizing by children to perceive the cultural-historical components of architecture}

In his growing trend from the beginning to the end, human beings have no similar learning capacities. The learning capacity of every individual starts to be formed from the childhood. Thus, all the periods of life of a person can be classified and studied with regards to memorizing. The childhood age that may be considered from the birth to the adolescence (puberty) is by itself including different growing periods that are classified and defined by different scientists. In that period and each growing stage, the child is susceptible to receive and learn specific categories of data. However, the question is which group among different age groups is susceptible to identify the components. By assessing the intellectual and personal capacities of the children in their different stages of growth, we shall clarify which group is considered by us to be in the top position of new learners of the history of architecture.

Here we look at the growth and stages of it from the perspective of three leading thinkers in this field.

\subsection{Erik Erikson Theory}

According to Erikson, human life has six different stages:

1) From the birth to one year of age (trust or distrust)

It is at this age that if the needs of a child are satisfied on time and the parents show their real sentiments to the child, he/she will find his/her world to be reliable and peaceful.

"Trust is developed via justification, continuity, and similar experiences in satisfying the main needs of the infant by the parents" (Bailer, 1999: p. 20). 
2) 2 - 3 years of age (independence against indecision)

"If the child is permitted and encourages doing what he/she can by his/her own ways and capabilities, the sense of independence will be created in him/her. If the parents are not patient and do anything instead of the child, he/she will doubt about his capabilities in confronting with the environment' (Bailer, 1999: p. 21).

3) 4 - 5 years of age (creativity against feeling guilty)

The language and physical activities of the child are challenged at this age; i.e. the activities that can raise the sense of creativity and responsibility in the child. The capabilities in participating in the physical activities and use of language will provide the basis for creative actions.

"Creativity adds another feature to the independence, which is responsibility, the feature for planning and pushing for doing a task only for being active and motivated" (Bailer, 1999: p. 21).

The danger of this period is in the sense of incompetence in reaching the aims that the person is always thinking about and the actions that are emerged due to passion towards the new moving and intellectual powers. If the child is left with freedom and initiative and if the parents spend enough time to answer his/her questions, the inclination for further discoveries will be developed in him/her.

4) 6 - 12 years of age (formation of constructive senses)

At this age, the child confronts with his/her environment more than before, affecting it and being affected by it. In fact, the child performs constructive action for the first time at this age, and if he/she is ignored and the feeling grows in him/her that his activities and questions are not considered or are frustrating, he/she feel guilty of doing a task in his/her own ways.

"The child should forget the past expectations and wishes, avoid extensive imaginations, and follow the environment regulations and even follow the required readings, writings, and calculations. At this stage, the person achieves the knowledge via creating the objects; i.e. he/she achieves constructive works. If the child is encouraged for constructing and executing various objects, and if he/she is permitted to finish what he/she has started and admired for his/her attempts, the constructive action will then start" (Bailer, 1999: p. 22).

5) 12 - 18 years of age (acquiring the identity)

The concept of identity is formed for the first time in the child personality. Playing a role in the community and evolving the concept of "self" are among the most important reconstructing factors of the identity.

"The growing and evolving adolescents, who face a bio-revolution from their inner side and also the tangible duties of adulthood from the outer side, refer their most important target to the point regarding the difference between the imagination of others from them and their own imagination from themselves. The adolescents searching for a new concept of identity and its continuity are forced again to challenge with the problems of the previous stages" (Bailer, 1999: p. 23). 
The aim in this stage is the evolution of the "self" identity; i.e. obtaining sufficient assurance from the homogeneity of the identity and its continuity. "The dander in this stage is the chaos of the role. If the adolescent is succeeded in integration of the roles in such a way that he/she is assured about perceiving himself/herself, the identity is then created. If he/she cannot be stable in different aspects of life, then the disorder in the role will be established.

6) Beginning of adulthood (association against seclusion)

This stage of growth is the important selection of the rate of intervention in the community or the seclusion.

"He/she is ready to associate with others. The danger in this stage is the possibility of close, competitive, and challenging relations experienced by the person for or against people that may result in his/her seclusion" (Bailer, 1999: p. 24).

Looking at Erikson classification, it can be inferred that capacities are emerged in the child during $6-11$ years of age and also $12-18$ years of age in the growing stages of the child, which provides a basis for receiving and perceiving the concepts such as the history of architecture. Although the points referred by Erikson are mainly considering the personal aspects of growth, dealing with the physical and mental characteristics of the person, but these characteristics can also be generalized to the social aspects of growth and formation of the child personality. In other words, the stages of construction and acquiring the identity do not only have the personal aspects in the growing trend and are also meaningful in relation with the outside world. For instance, by the age of $6-11$ and in the process of establishing the constructive feeling, the child tends to make relations with others and form his/her surrounding environment.

Accordingly, and also regarding the fact that architecture is essentially and intrinsically an affair that is mixed with creation and construction, this period of the growing stage can be considered as an important period in learning the architectural instructions (Ghayoumi Bidhindi, 2006). Although expressing the details of this relation and more precise proof of this claim requires a separate detailed study, but finding out the sensitivity of this age range in the formation of the sense of construction in the person (the child) and considering it as an opportunity for the instruction of the history of architecture are important issues to be regarded. The next age range introduced by Erikson (i.e. 12 - 18 years of age), the important subject of acquiring the identity is again considered. In fact, the child starts to acquire his/her identity in that period (by the beginning of the age of 12), and in case of disorders in the proper formation of this phenomenon, chaos and confusion of the role will emerge in his/her personality. This is the important issue in this study since the subject of identity is a cultural subject from the viewpoint of history and the history of architecture. In fact, according to the previous statements, the subject of "identity" with respect to the "history of architecture" is meaningless out of the issue of culture. Thus, a considerable part of the identity acquirement of the child at this age depends on the state of confronting with the cultural issues such as the history of architecture. It is clear that if the child has a proper and defined confrontation with the cultural phe- 
nomenon risen from his/her life (including architecture), he/she will have more profound and stable knowledge of the phenomena, having more opportunity in future for reviewing about, deep consideration to, and developing his/her cultural views.

"According to Erikson, the perception of the child from himself herself and from the culture that hel she has grown in is important as the sexual drive expressed by Freud"(Bailer, 1999: p. 25).

\subsection{Theory of Jean Piaget}

One of the other scholars, who have dealt with the subject of growth and its different stages, is Jean Piaget. It is no exaggeration if it is stated that no one has dealt with, observed and analyzed children's thoughts and behaviors as perfect and profound as Piaget.

According to Piaget, the growing stages of a child are as follows:

1) Sensorimotor stage (from the birth to two years of age)

According to Piaget, at this stage, learning the properties of objects is provided in the child via the senses and motor activities, and the completion of new ways for confrontation with the situations.

2) Preoperational stage (2 - 7 years of age)

At that period, the child is occupied with mental interventions in the symbols and objects, trying to identify his/her surrounding environment more than before. In other words, the first capabilities for recognizing the environment and the interventions (mental) in it are formed at that stage of life in the child.

3) Objective operation stage ( 7 - 11 years of age)

Identifying the objectives or the objectivity of the phenomena or items is formed at this age in the child. Capability in mental preservation (recording the phenomena) and memorizing them (often for using them in other situations in life)-decentralization or thinking simultaneously to more than one subject-are among the capabilities that are acquired by the child at this age.

"Piaget uses the concept of operation for explaining the state of acquiring the mental preservation. He defines the operations as the intrinsic interaction that changes the mental structure. The most important feature of the operations is their revocability, i.e. knowing that the current situation returns or can be returned to its origin" (Bailer, 1999: p. 26).

" $A$ while ago, the child was in the preoperational stage, not being able to do the justifications such as returning. The operation that an elementary school student can do is limited to the objects that are either in his possession or experienced directly and objectively by him/her. Thus, Piaget has named this stage as the objective operation stage. The child cannot do any superficial actions to deal with abstractive concepts, until he/she is not grown sufficiently and reached a better organization and conformity (usually by the end of the elementary school)" (Clark, 2006: p. 20).

Children do not usually reach to the perfect perception for the mental and capacity preservation until the age of nine or twelve. This indicates that the child 
requires more organization and conformity of the experiences before dealing with generalization of the principles.

4) 11 years of age and older

According to Piaget, this age is the age for imagining the future, considering the probabilities, and hypothesizing. In fact, the child or the adolescent can relate with the intangible world and the abstractive phenomena at this age, and think about them. The points regarding which phenomenon can cause the other phenomena, and what the causes of some affairs are, or what the roots of the required surrounding environment and the objects are and how they work, and what they are used for are all among the capabilities that are emerged at this age.

"Learning the abstracts will provide extraordinary extension to the person's horizon of thoughts. The adolescent can think about the appropriate perfection, discuss about the subjects that are in contrast to the reality, and present the required hypotheses. When the adolescent acquires more experience in superficial activities, he/she can come across more possibilities and pays less attention to the realities. Although the dominance of the adolescent provides the possibility for him/her to get used to more diverse matters and flexibility in his/her thoughts, but the dominance may also cause difficulties, since the adolescent can then analyze the positive and negative aspects of different future possibilities, such as selecting a profession that may be considered as an important anxiety resources" (Hilgard \& Bower, 1992: p. 57).

There are important points in the theory of Piaget about the last two age stages. Where he talks about the concept of memory for $7-11$ years of age, indeed, he considers the type of intellectual potentials that transform the mental structures of the child and hence, his/her negligence regarding the realities of the surrounding world. In other words, the analyzing mind starts working in that point and the primary inferences about the outer world are formed on the basis of the inner analyses of the child's mind. This is another evidence that in fact the mental capability in children in this period, both according to Piaget and also other scholars, is such that it is considered as the first age period in human life for understanding and receiving the objective and then the abstractive concepts. In continuing and stating about the features of the next age stage, i.e. over 11 years of age, Piaget expresses that the potential for learning the abstractive concepts are led to the extension of the thinking horizon, providing the possibility for the person to think about the appropriate perfection.

On the other hand, this age is important for the emergence of the perceiving potential of the real points as compared to the unreal ones and also the capability to establish various hypotheses. This age provides a considerable opportunity for learning the original concepts and acquiring the identity, which make the main intellectual aspects of the person, causing him/her to identify himself/herself in the world as well as his/her position. Recognizing the objective shapes in the living environment, such as the hose, the city, and the land, and recognizing the history of culture and traditions of the ecosystem, as the intel- 
lectual and abstractive cognitive forms can all be presented to the person in this period of life.

In another place, Piaget has pointed to the subject of choosing the profession, which may happen in this age, or at least the sparks of affinity or dislike may emerge in the person from a specific profession. Although Piaget mainly states the intellectual and spiritual effects of this phenomenon in the person, but the subject can also be looked at in a different aspect.

Being enthusiastic in a specific profession will lead to being interested in the tools, equipment, and the basis of learning and dealing with the profession, which is an important point to be considered. In fact, the child or the adolescent is quite interested to see the different angles and aspects of the profession that he/she is willing to do, in order to know it more properly. If the willingness or the motivation is conducted in an appropriate way, it can direct the adolescent person in his/her advancing trends regarding his/her interest to transform the coming years into developing his expertise.

On the other hand, looking into the activities in the surrounding world as a profession, even if it does not lead to selecting that profession by the person, will be the basis for him/her to work in that profession as the activity that some people are occupied with in specialized manner and understand the personal and social aspects of the activity in a more proper way. This can lead to realizing other fields of sciences and professions and can conduct the person in a better life in his/her future.

\subsection{Theory of Jerome Bruner}

Jerome Bruner is another scholar, who has explained the sequence of the growth stages, giving a proper instructive program in that basis, as he once stated that:

"Lack of a theory in instructions that can be used as a guide in the aspect of education is quite tangible. The theory of growth should be related with the theory of knowledge and the theory of education. Otherwise, it will not survive" (Hilgard \& Bower, 1992: p. 90).

The model of Bruner growth trend is in conformity with the model presented by Piaget in some parts, but it is also in contrast to that in some other parts. Regarding our considered age (the student of the second course in elementary school), Bruner believes that:

The student in the second course of elementary school reaches to the symbolic ideology from the visual ideology. Bruner also emphasizes on the general changes considered by Piaget, but he states then in another manner: The intellectual growth via increasing the child ability in simultaneous confrontation with different solutions, paying attention to different simultaneous sequences and allocating the required time and attention properly are defined by these various actions" (Shoarinejad, 1995: p. 85).

In this case, the age that is the age of reaching to the abstractive ideology is agreed between the scholars. However, the disagreements are about accelerating 
the thoughts of children in reaching the abstractive level. In fact, the disagreement here is due to the state of confrontations and training the children. Accordingly:

"Based on the viewpoints of Piaget, Alkind, and Ginsberg, it would be better to allow the children to gain their experiences in their own ways, and the attempts for accelerating the abstractive ideology is probably useless, confusing, and causing distress. However, Bruner and other insist that intensive attempts should be made for the students in the last years of the elementary schools to reach to the symbolic ideology by asking questions and making the required analyses. Bruner suggests that the teachers should organize the situations for the students to be encouraged to ask about the required phenomena (e.g.: asking 20 questions) and make hypotheses about the states of the definite events" (Singer \& Ronson, 1997: p. 35).

\subsection{The Researches about the Children Growth in Iran}

In addition to the viewpoints stated from the Western scholars regarding the children of this age, there are studies that are fulfilled in Iran, which can be useful. In some study for the existing packages, the children with different age groups are classified under the grade that they are studying. Our considered children are in the $4^{\text {th }}$ and $5^{\text {th }}$ classes or a little lower or more. In this studies, the students in each class (each age group) are undergone the field studies, and the results of the investigations reveal some other features for the age group.

1) Being interested in group works

The children of this age experience their first serious inclinations for participation in a group, and use it for further actions.

"The children in this age group enjoy group works, since they have shown great inclinations for continuing their tasks. The group cooperation improves their ideological processes" (Emad Khorasani, 2007: p. 53).

2) Being interested in reading books for finding the answers to the questions

Looking for researches to find the answers to the questions is emerged for the first time at this age.

"The $4^{\text {th }}$ grade students naturally become familiar with the library and its importance more the $3^{\text {rd }}$ grade students. On one hand, library as the important tool of the research becomes meaningful for them, and on the other hand, they become familiar with the concept of research that is trying and searching for acquiring new information" (Emad Khorasani, 2007: p. 60).

The morale for the group work, which was earlier described, helps the feature and prepares the children for considering group researches and finding the answers within a teamwork basis.

3) Accurate observation and using the past experiences in researches

This feature is mainly considered for the $5^{\text {th }}$ grade or higher level students.

"With what they have learned and the experience they have gained, the $5^{\text {th }}$ grade students are prepared to go to higher level in the official education, i.e. the course after the elementary school (guidance school or the first course of high 
school). They became familiar with the concept of two words, i.e. accuracy and asking, in the previous years, noticing that observing and then studying are among the important tools in researches. They also found out that they can search the answers to some of their questions in references and suitable books (Emad Khorasani, 2007: p. 53).

The established basis in educating the children of this age causes them to know about the research works more seriously, since they have gradually learned that:

"They should observe everything that is in their surrounding environment with great care. Observing indicates the use of the five senses (eyesight, hearing, smelling, tasting, and touching); i.e. whatever they see or hear should absorb their attention. They can collect some information about their living environment in this way. By observing, they find out the things that they should ask about. In fact, researching starts with different questions. These types of questions that they do not know the answers or they are not sure about the answers force them to ask and search for finding the answers.

Sometimes, a question may arise for them due to an observation, and a possible answer may also be given, which may not be right. Thus, they should search to find out whether the answers are correct or incorrect. In fact, they make a hypothesis in their mind according to their guess and they get the required answer though studies and searches in different books in the school library, or other appropriate and available books taken from public, the youth, or personal libraries, etc.). They require information about the subject they have chosen according to their own ideas and using the ideas of the other members of the family and also the available public media (radio, television, newspapers, etc.), and they should use reference books and readable materials and do the related searches by the instructions of the librarians, to obtain the required information. They should take notes about the specifications of books and other readable materials, and they should represent the results of their observations, the answers from the asked questions (via interviews) and their research as a whole in different ways such as speeches, drawings, and writing simple sentences" (Emad Khorasani, 2007: p. 53).

The students in that age range can do their research works in individual or group basis. Moreover, they have found that they can extend the level of their ordinary library studies and enter into the process of creativity and initiative.

During the process, they are prepared to use the library and media sources as the basis for their information literacy, and they can guess about the answers to the considered questions. On the other hand, they are ready to test and measure their viewpoints and guesses in order to specify the correct or incorrect answers.

\section{Research Method}

Research method is based on historical, descriptive, analytical, and field study methods. In terms of theoretical debates, analytical and descriptive methods use 
based on provided information in library documents of different organizations. In the next stage, to do this research, we need two areas of recognition: one recognizing the world of children's education (in terms of recognizing practices and tools) and the other recognizing understandable doctrines of architectural history for children. The way we enter these discussions is, first of all, an audiences that it examines the methods and tools of child education. After gaining the necessary knowledge of this topic, we explore various types of architectural history tutorials, which explain them to the most appropriate of them by adapting to the methods of teaching children. The result of such a process is that it becomes clear at first whether children can reach the audience the architectural history tutorials, and secondly, if so, what can be achieved by this familiarity.

\section{Finding}

Conformity of the learning capacity in children with the perception of the instructions for the history of architecture

According to the above mentioned points about the capacity of learning in children, a considerable part of the architectural history instructions can be presented to the children in the defined ages, and they are somewhat linked to potential that the children have in learning. The aim of the current study is not presenting the learning contents for the children and providing the solutions as well as compilation of the contents should be used in other and independent studies.

According to the above, the relation regarding the identification of the history of architecture and the intellectual capacities of the child from 7 years of age to the age of adolescence can be described within the predicate reports. Briefly speaking, the result of the viewpoints of the scholars in the field of children growth regarding the capabilities of the children in comprehensions and relating with the surrounding world are as follows:

- Excessive confrontation with their environment

- The first step for construction

- Formation of the concept of identity for the first time

- Identification of the objectivities or the superficial objectives of the objects or phenomena

- Attention in observing the capability in mental preservation (memorizing the phenomena) - using them in other situations in life

- Decentralization or simultaneous thinking to more than one subject

- Imagining the future-considering the probabilities and hypotheses

- Relating with the intangible world and abstractive phenomena

- Tracking the causes of the phenomena-finding the origins regarding what the surrounding phenomena are and what the objects and tools for them are, and how they work

- Interests and inclinations towards group work

- Being interested in books and reading them to find the answers to the re- 
quired questions

Each of the above topics, as described as follows, is related to recognizing and the knowledge of the history of architecture and its components.

\subsection{Excessive Confrontation with their Environment (6 - 11 years of Age)}

(An approach for recognizing architecture as a great part of the surrounding environment)

In accordance with the viewpoints of the above experts, the child is tangibly confronted for the first time at this age with his/her surrounding world. Thus, he/she would have the ability to identify the manmade environment as compared to the natural environment. As the most important and even generally, the only manmade environment, architecture can be identified by the child at this stage. At this age, the child becomes familiar with the concepts such as inside and outside, open and closed space, and old or new area, all of which make the alphabet for recognizing the surrounding environment. Clearly, an expert and systematic encounter for education and transferring the concepts to him/her can create a more profound and effective perception for him/her about his/her surrounding environment.

\subsection{The First Step for Construction}

\section{(Recognizing architecture as the constructed space)}

One of the most important events at this growing stage for children is with regards to the concept of construction and building. At this age, the children find out that they can intervene in their surrounding environment via construction. They also realize that many of their surrounding phenomena are man-made, which are constructed by various stages (Polsco, 2016: p. 85). Architecture can also be introduced to the children as a constructed space for living and human activities. At this age, the children can realize why and how humans provide architecture of their surrounding environments. The answers to these questions can easily and comprehensibly describe the effective factors on emergence of an architectural work and the required motivations to the children. Moreover, the child can identify the objects via exercises, tests, or his/her own experiences and tangibly realize the subject.

\subsection{Formation of the Concept of Identity for the First Time (from 12 Years of Age to Adolescence)}

(Introducing architecture and the living area as the element for acquiring the identity

One of the main stages for the intellectual growth of children is the formation of their identity in this period. At this stage, the child gradually finds himself/herself in the world that he/she was born. The important point here is the education of the child in the direction for him/her to become acquainted with his/her own cultures in order to organize and develop his/her position and iden- 
tity in proper and stable basis. Familiarity with the history of architecture in a land such as Iran, with its rich background in architecture, can definitely introduce the aspects of his/her life, relatives, and ancestors more appropriately. Thus, the child would realize that the house, working place, entertaining area, or any other spaces, where he/she is involved with and work in, is the inseparable part of his/her life, considerably affecting his/her behavior and form his/her personality.

\subsection{Identification of the Objectivities or the Superficial Objectives of the Objects ( 7 - 11 Years of Age)}

(An opportunity to confront with the objectivity and form of architecture)

Architecture is one of the visual arts, and it is intrinsically a tangible and apparent affair. Thus, it is both by creating a work and also by recognizing and perceiving that, it clearly has an extensive role. Since the first capabilities for the objective confrontations with the phenomena are formed in this age in children, their minds try to observe and record the architectural works. This opportunity is considerable, since recording the objectivities in children's minds can occur in a more stable and durable basis.

In addition to observing the architectural works, the child pays attention to the similarities and differences, and the potential to record and classify the architectural works is created according to the appearances. Moreover, the child can express his/her viewpoints and feelings about the appearance of the works, and explain the required reasons. The confrontation with the appearance of the works and feeling them closely are perhaps the most durable and effective experience that happens on the field of architecture in the child's mind, providing more affection of him/her with the history of architecture.

\subsection{Attention in Observing, Capability in Mental Preservation and Memorizing the Phenomena, and using the Experiences in other Situations in Life (11 Years of Age and Older)}

(Accomplishment of the capacity of recording the events and using the subjects of the history of architecture for future)

The ability to record the phenomena and events in the mind is related to learning the history of the phenomena such as architecture. By the accomplishment of this capacity in children, they obtain the required backgrounds for familiarity with the history of various phenomena and can realize the sequential and continuous events. In fact, the emergences of the capability to record the events mainly help the theoretical aspects of architecture including the history of architecture and the cultural events. This capability is quite important due to perceiving the classifications and categorization of architectural works or the periods of historical architecture. At this age, the children can become familiar, for the first time, with different types of architectural works and their similarities or differences, and classify them in their minds with regards to various aspects.

However, the other skill that the children acquire at this age is using their own experiences in other affairs of life. Earlier on, it was stated that one of the most 
important advantages for studying history in general form, and the history of architecture in specific form, is using the experiences for building the future. Thus, the children at this age can realize that architecture has a past, a present, and a future, which are linked together hierarchically, and the obtained experiences in the past should be used as the guidance for future.

\subsection{Decentralization or Simultaneous Thinking to More than One Subject (7 - 11 Years of Age)}

\section{(Ability to think about architecture as a phenomenon that is derived from} various factors)

Polygonal aspect of the history of architecture and its dependence to various cultural, political, and economic factors have caused the better perception of architecture also have polygonal requirements. In fact, some forming and effective factors should simultaneously and in interactions with each other be considered for better understanding of architecture. Emergence of simultaneous thinking about various subjects will provide the possibility in children to learn various effective elements on architecture, in basic form, and think about the relations between the required elements.

\subsection{Imagining the Future-Considering the Probabilities and Hypotheses (11 Years of Age and Older)}

\section{(Imagination and visualization of the future of architecture)}

Regarding the existence of the capacity of thinking about future, imagining the future of the architectural works and the shape of the cities based on what the children have learned from the past and history of architecture will be attractive for them at this age. This ability that is mainly of the form of imagining the appearance of architectural works or the shape of houses and cities can form the future of architecture in the child's mind, even prematurely and imaginatively. Along with the superficial imaginations, children can also deal with hypothesizing or telling stories about what may happen in the future, and talk about them or even write about them and draw them. Proper use of this capacity can develop the sense in children that history is a continuous string and we will intervene about what will happen in future.

\subsection{Relating with the Intangible World and Abstractive Phenomena (11 Years of Age and Older)}

\section{(Ability to perceive the intangible and effective factors on life)}

Most of the factors forming the style of life and effective on the emergence of architectural works are intangible and abstractive factors; the factors such as worldviews and beliefs, religion, literature and the myths and many other similar affairs. In his/her 7 years of age up to puberty, the child finds out about the existence of such factors in life, tending to recognize them due to his/her curiosity. This capacity and curiosity provides the possibility for the child to perceive the roles of the intangible factors in the emergence of architecture and relate them 
with each other.

\subsection{Tracking the Causes of the Phenomena and Finding the Origins of the Affairs (11 Years of Age and Older)}

(Required capacity for perceiving the causes of the emergence of architecture and the causation relations of the architectural works with their styles)

At this age, the children learn that every phenomenon in the surrounding world has a related cause, and architecture is not an exception in this regard. Accordingly, the children can ask about the reasons for the emergence of architectural works, learn about the advantages of reading the history, and ask about the shapes and facades of the constructions and the reasons for such appearances. By relating between the causes and effects in the history of architecture, the children learn how to influence the effects (architectural works) by changing the causes, and learn about what factors affect the transformations in architecture specific periods, and also about the factors in the architectural stabilities and developments. However, these concepts should be translated into the children's language by the specialists in the field of education to be presented to them in basic and comprehensive form.

\subsection{Interests and Inclinations towards Group Work (10 Years of Age and Older)}

(The experience of construction by group work and familiarity with architecture as the practical group work)

By the formation of the primary inclinations towards group work, the children begin to enter larger communities for executing different activities and recognizing the surroundings. At this age, the children experience group work directly and can also become familiar with the group activities of others. Architecture is among the activities that depend on collective work, and it is the result of many people with different specializations. This has been an affair that has always existed during the history and not only related to the modern architecture. At this age, the child enjoys the potential to perceive the subject. This feature along with the construction ability, as described earlier, can form a great part of the child's recognition about the history of architecture.

\subsection{Being Interested in Books and Reading them to Find the Answers to the Required Questions (10 Years of Age and Older)}

(Created capacity for familiarity with the history of architecture via studies and researches)

Knowing the history of architecture is not only resulted via observing the appearance of architectural works and confrontations with the appearances. The great part of this recognition for any addressee will be definitely done via studying. Recognizing a considerable part of the history of architecture is only possible via studies. The main resources for knowing the history are formed by the historical contexts. In other words, the main resources in recognizing the history 
of architecture are the historical and up-to-date books and contexts. The components such as worldviews and beliefs as well as the history of the ancient tribes and also the knowledge of geography, ecology, and their related pints in the field of architecture are obtained via studying specialized books and contexts. However, it is not intended to impose the studying of the specialized contexts in these fields to the children, but whatever is issued in simple language for the children is definitely useful for them.

\section{Conclusion}

It can be concluded from what was stated herein that the components existing for the recognition of the history of architecture can be described to the children, although they do not intrinsically have similar nature. This potential is due to the emergence of the intellectual capacities in children with specific age, which was considered earlier. Since by the beginning of its formation, the child's mind has prominent potentials in learning and memorizing the events and the data, confrontation with the concepts of the history of architecture and familiarity with the architectural culture can be quite useful for him/her. Moreover, this familiarity is one of the best ways to prevent the cultural breakdown and the resulted alienation in future generations.

Generally speaking, the intellectual potentials of children from the viewpoint of the experts, whom according to the present study were related to the knowledge and perception of the history of architecture, were considered within the framework of 11 predicates. Each of these 11 aspects is somehow related to the cognition and knowledge of the history of architecture, the relation of which was described within this chapter. The children can be considered to be susceptible for the perception of the instructions regarding the history of architecture in accordance with that relation.

\section{References}

Bailer, R. (1999). Psychology Applied to Teaching (6th Edition, pp. 15-255). Tehran: University Publication Center Press.

Clark, M. (2006). Writing for the Children (pp. 20-100). Tehran: Cultural Research Office.

Emad Khorasani, N. (2007). Research on the Elementary School Students (pp. 5-80). Tehran: Cultural Research Office.

Ghayoumi Bidhindi, M. (2006). Architectural Instructions in Pre-Modernism Era Based on Resaleh Memarieh. Soffeh, No. 42, 65-85.

Hilgard, E., \& Bower, G. (1992). Learning Theories (2nd Edition, 40-130). Tehran: University Publication Center.

Polsco, D. (2016). Art in Children's World (pp. 10-110). Tehran: Shahid Rajaei Teacher's Education University Press.

Singer, D. J., \& Ronson, T. A. (1997). How Does a Child Think? (pp. 12-150). Tehran: Amouzesh Publications.

Shoarinejad, A. A. (1995). Psychology of Growth (pp. 10-200). Tehran: Etelaat Publications. 